\title{
Calculation of magnetic devices cleaning coolants in the agro-industrial complex
}

\author{
Boris Avdeyev ${ }^{1, *}$, Victor Prosvirnin ${ }^{1}$, and Roman R. Dema ${ }^{2}$ \\ ${ }^{1}$ Kerch State Maritime Technological University, Ordzhonikidze st., 82, Kerch, 298309, Russia \\ ${ }^{2}$ Federal state Budgetary Institution of Higher Education "Nosov Magnitogorsk State Technical \\ University” (FSBIHE "NMSTU”), Lenin Avenue, 38, Magnitogorsk city, 455000, Russia
}

\begin{abstract}
Traditional ways of cleaning of cooling lubricants in agrarianindustrial complex are considered in the article. Experimental data on the presence of magnetic impurities and its fractional composition as a result of parts machining on machines with the use of cooling lubricants are cited. Combined devices based on the combination of traditional methods for cleaning technical fluids have been used to intensify the cleaning process. The use of such combined cleaning methods is grounded, the forces effecting on the particles in the process of separation - coagulation forces, magnetic deposition, and medium resistance - are considered. The mathematical model of the coagulation process of two particles having similar size in a homogeneous magnetic field is made up. However, it is impossible to solve the obtained system even by a numerical method with unknown initial conditions. In this connection, the inverse problem is proposed. The physical significance of the effect of forces is as follows: a floccule consisting of two particles is destroyed under the effect of coagulation forces; the resistance of the medium prevents it. Its numerical solution allows finding the main dynamic characteristics of particle coagulation: the speed and distance from which they interact.
\end{abstract}

\section{Introduction}

In the agrarian-industrial complex, the cooling lubricants (CL) are used for machining tough steels and alloys, the complex mechanism of their effect comes both to a decrease in the processes of plastic strain of the metal, the roughness of its surface during machining, and to reduce of friction, wear and deterioration of the metal structure, as well as the instrument [1,2].

For example, waste generated in the process of grinding and polishing is $30-40 \%$ of the material of the grinding wheel, $60-70 \%$ of the material of the work piece [3]. The amount of evolved material depends on various parameters: the hardness of the material being processed, the diameter and circumferential velocity of the circle, the method of the product feed, etc. More than $50 \mathrm{~g} / \mathrm{h}$ of dust from one machine is emitted when processing products from steel. The fractional composition is shown in Fig. 1.

\footnotetext{
*Corresponding author: dirigeant@mail.ru
} 


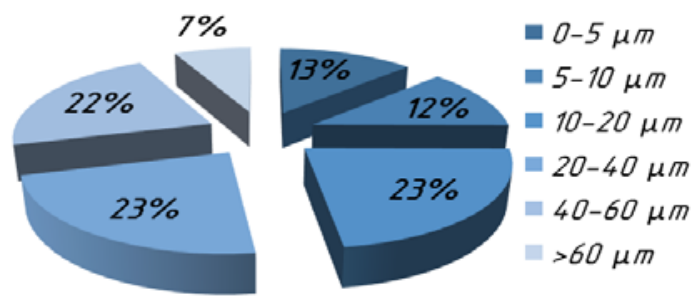

Fig. 1. The fractional composition of metal powder when grinding

The characteristic of the slurry in CL of the grinding section is shown in Table 1. The cooling lubricant cost in the agrarian-industrial complex is up to 4 times higher than the cutting tools cost and reaches $17 \%$ of the prime cost of manufacturing machine parts $[4,5]$.

Table 1. The characteristic of the slurry of the grinding section. is shown in

\begin{tabular}{|c|c|c|c|}
\hline \multirow{2}{*}{ The characteristic of the slurry } & \multicolumn{3}{|c|}{ Grinding type } \\
\cline { 2 - 4 } & black & semi black & clean \\
\hline Density, $\mathrm{kg} / \mathrm{m}^{3}$ & 4075 & 3700 & 3150 \\
\hline Medium diameter of particles, mm: \\
\hline metal & 0,8 & 0,65 & 0,5 \\
\hline abrasive & 0,5 & 0,4 & 0,32 \\
\hline Particle amount in slurry, \%: \\
\hline metal & 95,5 & 92,5 & 90,5 \\
\hline abrasive & 4,5 & 7,5 & 9,5 \\
\hline The amount of slurry (from one machine, $\mathrm{kg} / \mathrm{h}$ ) & 1,4 & 1 & 0,6 \\
\hline
\end{tabular}

Appropriate devices for purification of technical fluids from mechanical impurities, usually based on traditional methods, are envisaged to extend the resource indices in systems and installations [6,7].

Electromagnetic devices for purification of viscous media can be divided into the following main groups: coagulators, settling tanks, filters, filter separators and separators. In recent years, combined devices based on the combination of traditional and nontraditional ways for purification of technical fluids - the use of electrical fields (magnetic, electric, ultrasound, etc.) began to be used to intensify the purifying process [8].

\section{Material and methods}

Analysis of existing information and long-term researches has shown that combined methods have not been fully implemented yet. The main reason is the lack of scientifically based methods and recommendations for the calculation and implementation of various combined systems and devices for purification of technical fluid from mechanical impurities, which restrains the creation of new and improvement of existing purifying devices [9].

This allows us to draw general conclusions on the state of this issue:

- traditional methods and technical means have almost exhausted themselves;

- one of the ways to increase the degree of purification is the application of external influences in the form of electric fields;

- the overwhelming amount of mechanical impurities of cooling lubricants that arise during operation and during the repair of agricultural machinery, fully or partially relates to 
ferromagnets and allows intensification of purification by magnetic effect;

- the energy of the magnetic field being relatively small in absolute value can cause a significant increase in the degree of purification.

\section{Results and its discussion}

When particles get the operating space of the electromagnetic device in a magnetic field, they stick together, forming floccules of the acicular structure, which move towards the field points with the greatest intensity.

The extraction of particles from a dispersed medium with the forces of a magnetic field includes three types of motion: orientation (turning through force lines of the field elements of rotational motion); formation of floccule; precipitation of floccule and single (uncoagulated) particles.

Depending on the parameters of the particles, the medium and the magnetic field, the description of the motion of each individual particle is difficult due to the large number of calculations and the complexity of determining the initial conditions. The presence of connections between the moving particles does not allow us to examine them separately experimentally. However, each of the movements can be investigated on the basis of physical laws and regularities having strict mathematical formulations. These mathematical dependencies will give a general mathematical model for the extraction of ferromagnetic particles in appropriate devices.

The magnitude of the magnetic force effecting on the particle is calculated according to the expression:

$$
F_{M}=\mu_{0} \cdot \chi \cdot V \cdot H \cdot \frac{\mathrm{d} H}{\mathrm{~d} l}
$$

where $\mu_{0}=$ const $=4 \cdot \pi^{-7}, \chi$ and $V$ - magnetic perception and volume $\left(\mathrm{m}^{3}\right)$ of the particle accordingly; $H$ and $\mathrm{d} H / \mathrm{d} l$ - field strength $(\mathrm{A} / \mathrm{m})$ and rate of its change $\left(\mathrm{A} / \mathrm{m}^{2}\right)$ along motion of particles accordingly.

Moreover the particles (floccules) interact with each other with a force defined by the Coulomb's law for "magnetic masses" [10]:

$$
F_{F}=\frac{4 \cdot \pi \cdot M_{1} \cdot M_{2}}{\mu_{0} \cdot \mu_{f} \cdot R_{F}^{2}},
$$

where $M_{1}, M_{2}$ - "magnetic masses" of particles, $\mathrm{m}^{2} \cdot \mathrm{kg} / \mathrm{s}^{2} \cdot \mathrm{A} ; \mu_{f}-$ the magnetic permeability of the medium in which the particles are located; $R_{F}$ - the distance between the particles, $\mathrm{m}$.

When moving in viscous media, additional forces appear that prevent the movement of particles (floccules). The strength of the medium's resistance, according to the Stokes law, is:

$$
F_{D}=-3 \cdot \pi \cdot d \cdot \eta_{f} \cdot U_{F},
$$

where $\eta_{f}$-dynamic viscosity of viscous media, $\mathrm{Pa} \cdot \mathrm{s}$.

Influential parameters of magnetizing devices (solenoids, electromagnets, permanent magnets, etc.) by means of which coagulation and extraction of particles is carried out are the magnetic field strength $H$ and the magnetic susceptibility of the particles $\chi$, all other conditions being equal. 
Coagulation of particles is the most complex and insufficiently explored problem. Under certain conditions, it is primitive and dominates the general process of extracting particles from viscous media [11].

Let's assume that the magnetic field in the areas of the arrangement of the particles is homogeneous $(\mathrm{d} H / \mathrm{d} l=0)$, the effect of forces on them coincides with the direction of the field strength vector $\mathrm{H}$ (Fig. 2a). The magnetic deposition force of $F_{M}$ is zero. Without taking into account the gravity and buoyancy, the coagulation forces $F_{F}$ and the resistance of the medium $F_{D}$. will affect the particles. According to the second law of dynamics, the following equality exists for each of the 2 particles of mass $\mathrm{m}$ :

$$
F_{1}=m_{1} \cdot a_{1}=\overrightarrow{F_{D 1}}+\overrightarrow{F_{F 1}} ; F_{2}=m_{2} \cdot a_{2}=\overrightarrow{F_{D 2}}+\overrightarrow{F_{F 2}} .
$$

or in a differential form:

$$
m_{1} \frac{d \overrightarrow{U_{F 1}}}{d t}=\overrightarrow{F_{D 1}}+\overrightarrow{F_{F 1}} ; \quad m_{2} \frac{d \overrightarrow{U_{F 2}}}{d t}=\overrightarrow{F_{D 2}}+\overrightarrow{F_{F 2}} .
$$

The distance traversed by particles is calculated according to the expressions

$$
\begin{gathered}
\frac{d \vec{Y}_{1}}{d t}=\vec{U}_{F 1} ; \quad \frac{d \vec{Y}_{2}}{d t}=\vec{U}_{F 2} ; \\
m_{1} \frac{d^{2} \vec{Y}_{1}}{d t^{2}}=\overrightarrow{F_{D 1}}+\overrightarrow{F_{F 1}} ; \quad m_{2} \frac{d^{2} \overrightarrow{Y_{2}}}{d t^{2}}=\overrightarrow{F_{D 2}}+\overrightarrow{F_{F 2}} .
\end{gathered}
$$

Equations (6) and (7) describe the interaction of particles in a magnetic field.
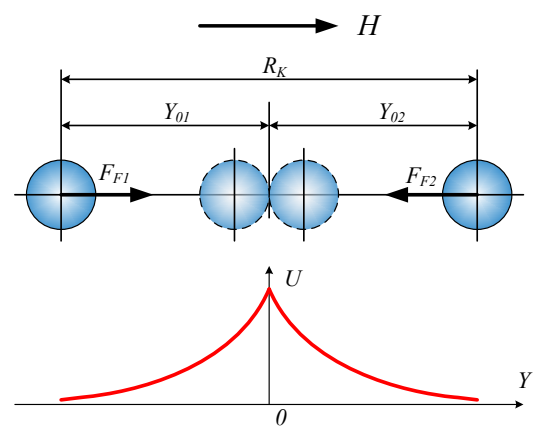

a)
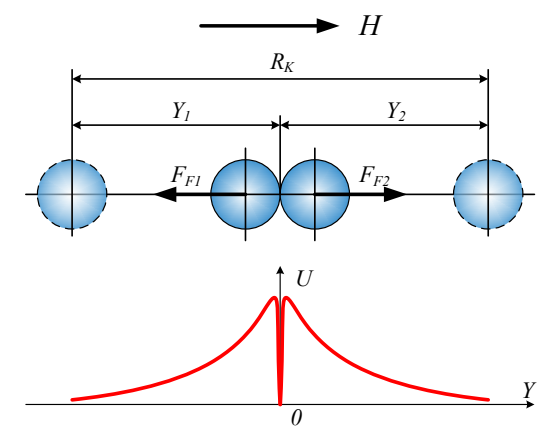

b)

Fig. 2. The mechanism of interaction (coagulation) of particles in a magnetic field: a) primal problem; b) the inverse problem

The magnetic coagulation force $F_{F}$, taking into account the "magnetic mass", is calculated according to following formula [12]:

$$
\begin{gathered}
F_{F}=\frac{\pi^{3} \cdot \mu_{0} \cdot d_{1}^{2} \cdot d_{2}^{2} \cdot H^{2}}{9 \cdot R_{F}^{2}}\left(\frac{\chi}{1+N \cdot \chi}\right)^{2} ; \\
R_{F}=\left(Y_{1}+Y_{2}\right) .
\end{gathered}
$$


It can be seen from expression (8) that the coagulation force depends on the magnetic susceptibility $\chi$ and particle size, the external field $H$, and also the distance between the particles $R_{F}$.

Substituting the values of $F_{D}$ and $F_{F}$ from (3) and (8) into the system of equations (7), taking into account the fact that

$$
\chi_{b}=\frac{\chi}{1+N \cdot \chi}
$$

где $\chi_{b}$-magnetic susceptibility of body, we get:

$$
\begin{aligned}
& -m_{1} \frac{\mathrm{d}^{2} Y_{1}}{\mathrm{~d} t^{2}}=-3 \cdot \pi \cdot d_{1} \cdot \eta_{f} \cdot U_{F 1}+\frac{\pi^{3} \cdot \mu_{0}}{9} \cdot \frac{d_{1}^{2} \cdot d_{2}^{2}}{\left(Y_{1}+Y_{2}\right)^{2}} \cdot \chi_{b}^{2} \cdot H^{2} \\
& -m_{2} \frac{\mathrm{d}^{2} Y_{2}}{\mathrm{~d} t^{2}}=-3 \cdot \pi \cdot d_{2} \cdot \eta_{f} \cdot U_{F 2}-\frac{\pi^{3} \cdot \mu_{0}}{9} \cdot \frac{d_{1}^{2} \cdot d_{2}^{2}}{\left(Y_{1}+Y_{2}\right)^{2}} \cdot \chi_{b}^{2} \cdot H^{2}
\end{aligned}
$$

When initial conditions $t=0 ; t(0)=0$.

$$
\frac{\mathrm{d} Y_{1}}{\mathrm{~d} t}(0)=U_{F 10} ; \frac{\mathrm{d} Y_{2}}{\mathrm{~d} t}(0)=U_{F 20} ; Y_{1}(0)=Y_{10} ; Y_{2}(0)=Y_{20}
$$

The system of equations (10) with the initial conditions (11) is a mathematical model of the process of magnetic coagulation of two particles similar in size in a viscous medium. Solution (10) and (11) with known values of the parameters of the particles and the medium will allow the conditions for the formation of floccules, and hence the initial data for calculating the magnetic field $[13,14]$.

However, as follows from (8), the action of the force $F_{F}$ is endless in space, i.e. particles being in the field, always have effect on one another, thereby surely moved, having the defined distance values $Y_{1}$ and $Y_{2}$ and defined speed values $U_{1}$ and $U_{2}$ in any given moment of time. Therefore, it is impossible to solve system (10) even with a numerical method with unknown initial conditions (11).

To solve system (10), we consider two ferromagnetic particles, on which the forces $F_{D}$ and $F_{F}$ effect, as shown in Fig. 2 b. In this case, the physical significance of the effect of forces is reduced to the following. The floccule, consisting of two particles, is destroyed under the effect of $F_{F}$, force $F_{D}$ interferes it. At the beginning of motion path at $\mathrm{t}=0, F_{F}$ has a maximum value, $F_{D}$ is zero, so the motion of the particles in opposite directions is accelerated. As the distance between the particles increases, $F_{F}$ decreases, while the force $F_{D}$ increases due to the increase in the velocity $U_{F}$. At moment of time $t=t_{1}$, the forces $F_{F}$ and $F_{D}$ tend to a maximum. With further motion of the particles, $F_{F}$ and $F_{D} t_{\text {end }}$ to zero and at the time $t=t_{F}$ forces $F_{F}, F_{D} \rightarrow 0$. Thus, during a time $0 \ldots t_{F}$, the particles go away to a distance $R_{F}$. The dynamics of the particles will then be described with the same differential equations (only the signs in front of the terms change) with known initial conditions:

$$
\begin{aligned}
& \frac{\mathrm{d}^{2} Y_{1}}{\mathrm{~d} t^{2}}=-A_{1} \cdot \frac{\mathrm{d} Y_{1}}{\mathrm{~d} t}-D_{1} \frac{\chi_{b}^{2} \cdot H^{2}}{\left(Y_{1}+Y_{2}\right)^{2}} ; \\
& \frac{\mathrm{d}^{2} Y_{2}}{\mathrm{~d} t^{2}}=A_{2} \cdot \frac{\mathrm{d} Y_{1}}{\mathrm{~d} t}-D_{2} \frac{\chi_{b}^{2} \cdot H^{2}}{\left(Y_{1}+Y_{2}\right)^{2}} .
\end{aligned}
$$

At $t=0: t(0)=0$ : 


$$
\begin{aligned}
& U_{F 1}(0)=U_{F 10}=0 ; \quad U_{F 2}(0)=U_{F 20}=0 ; \\
& Y_{1}(0)=Y_{10}=\frac{d_{1}}{2} ; \quad Y_{2}(0)=Y_{20}=\frac{d_{2}}{2}
\end{aligned}
$$

where

$$
A_{1}=\frac{18 \cdot \eta_{f}}{d_{1}^{2} \cdot \rho_{p}} ; D_{1}=\frac{2 \cdot \pi^{2} \cdot \mu_{0} \cdot d_{2}^{2}}{3 \cdot d_{1} \cdot \rho_{p}} ; A_{2}=\frac{18 \cdot \eta_{f}}{d_{2}^{2} \cdot \rho_{p}} ; D_{2}=\frac{2 \cdot \pi^{2} \cdot \mu_{0} \cdot d_{1}^{2}}{3 \cdot d_{2} \cdot \rho_{p}} \text {. }
$$

$\rho_{p}$ - the particle density, $\mathrm{kg} / \mathrm{m}^{3}$.

\section{Conclusions}

The process of coagulation of magnetic particles plays an important role in the process of cooling lubricants in the agrarian-industrial complex. The developed system of differential equations with initial conditions allows us to calculate the main dynamic characteristics of particle coagulation: the speed $U_{F}=f\left(t, R_{F}, d, p_{p}, \eta_{f}, \chi_{b}, H\right)$ and the distance from which they interact $R_{F}=f\left(t, U_{F}\right)$, which is necessary for the designing of systems and cleaning devices.

The work is fulfilled under the financial support of the Ministry of Education and Science of the Russian Federation, project no. 11.2054.2017/4.6 under the state task for 2017-2019.

\section{References}

1. V. Masyagin, R. Bazhenov, A. Muholzoev, MATEC Web Conf.: Int. Conf. on Mod. Trends Manuf. Technol. Equip. 129, 01063 (2017)

2. S. Chernyi, IOP Conf. Ser.: Mater. Sci. Eng., 124 (2017)

3. E.S. Kiselev, V.N. Kovalnogov, Scientific bases and technology of application of lubricating-cooling technological means at machining (Ulyanovsk, UISTU, 2008)

4. S.I. Platov, R.R. Dema, M.V. Kharchenko, R.N. Amirov, Experience of application of liquid lubricating materials during wide strip hot rolling 3rd International Sc. and Technic. Conf. on Sc. and Technical Progress in Ferrous Metal. (2017)

5. B. Avdeyev, MATEC Web Conf.: Int. Conf. on Mod. Trends Manuf. Technol. Equip. 129, 06012 (2017)

6. V.A. Zhukov, E.P. Masyutkin, B.A. Avdeyev, IOP Conf. Ser.: Mater. Sci. Eng., 177 (2017)

7. B. Avdeyev, E. Masyutkin, S. Golikov, S. Sokolov and V. Gavrilov, Calculation of efficiency curve of magnetic hydrocyclone 2017 IEEE Conference of Russian Young Researchers in Electrical and Electronic Engineering, pp. 1225-1228 (2017)

8. S. P. Srivastava, Developments in Lubricant Technology (Wiley-Blackwell, 2014)

9. E.T. Bulyzhev, E.N. Men'shov, Mathematical theory of magnetic coagulation of ferromagnetic particles in weakly contaminated sewage Izvestia Samara Sci. Center Russ. Acad. Sci., 16 1, pp. 247-254 (2014)

10. S.V. Vonsovsky, Magnetism. Magnetic properties of dia, vapor, ferro, antiferro, and 
ferrimagnetics (Science, Moscow, 1971)

11. A.A. Kamenskaya, R.I. Kovalova, V.M. Labetsky, The impact of metal processing industries by cutting machine-building enterprises on the environment and ways to reduce the damage caused (Novosibirsk, 2002)

12. V. Budnik, S. Chernyi, Procedia Eng., 150, (2016)

13. A.A. Sokolov, A.S. Miroshnikov, E.A. Sokolova, Gornyi Zhurnal, 12, 83 (2016)

14. E. Sokolova MATEC Web Conf.: Int. Conf. on Mod. Trends in Manuf. Technol. and Equip. 12903008 (2017) 